\title{
CORRIGENDUM
}

\section{The effect of Hus1 on ionizing radiation sensitivity is associated with homologous recombination repair but is independent of nonhomologous end-joining}

\author{
X Wang, B Hu, RS Weiss and Y Wang
}

Oncogene (2008) 27, 1343; doi:10.1038/onc.2008.1

Correction to: Oncogene (2006) 25, 1980-1983. doi:10.1038/sj.onc.1209212; published online 7 November 2005.

a
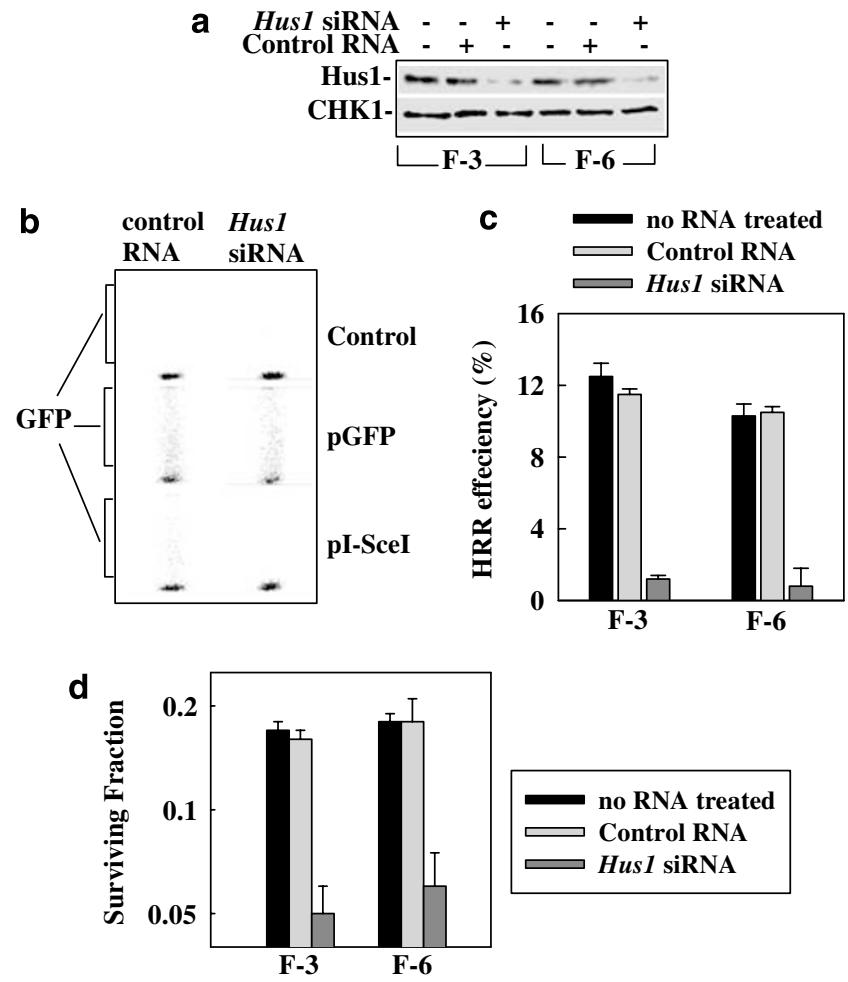

Since the publication of the above paper, the authors have identified an error in Figure 3a. The revised version of the figure is given here.

Figure 3 Reduced Hus 1 expression impairs HRR. (a) The levels of Husl expression were measured with the whole cell lyses from either Hus1 siRNA or control RNA treated F-DRGFP cells. Hus1 antibody was prepared as follows: a Hus1 fragment (Huslf, 606$846 \mathrm{bp}$ ) from mRNA of NIH3T3 cells was amplified by using RTPCR. The PCR products were inserted into pET-28a $(+)$ vector; the plasmid was transformed into host cell BL21(DE3) for the Huslf expression. The expressed protein was purified by chelating column (Ni-NTA, Qiagen). Polyclonal antibody was made by immunizing mice using the purified protein and was confirmed by Western blot to recognize the Husl fragment protein specifically. Husl siRNA that specifically targets the sequences of the mouse Hus1 mRNA (5'-CCUGCACCCUCCGCAUCAGUU-3') was designed. The designed siRNA was synthesized by Dharmacon Company. The Scrambled RNA (Dharmacon Company) was used as the transfection control. The transfection of siRNAs was performed with the oligofectimine (Invitrogen) following the manufacturer's instructions. The cells were prepared for further examination (HRR, western blot and IR) at $48 \mathrm{~h}$ after transfection. Western blotting was performed with whole cell lysates using antibodies against Hus1 and CHK1 (sc-8404, Santa Cruz Biotechnology, Inc.) as described (Hu et al., 2005). CHK1 was used as the internal loading control. (b) Measurement of HRR was as described (Hu et al., 2005). Representative flow cytometric analyses of GFP signal in F-3 cells following DSBs. The cells were either transfected with pGFP (alone or cotransfected with siRNA) or pCMV3xnlsI-SceI (pI-SceI) (alone or cotransfected with siRNA) with Lipofectamine 2000 (Invitrogen) following the manufacturer's instructions. The cells were then transfected with either Hus1 siRNA or control RNA by using the oligofectimine (Invitrogen) following the manufacturer's instructions at $24 \mathrm{~h}$ after the first transfection. The cells were collected at $48 \mathrm{~h}$ following the siRNA transfection. (c) HRR efficiency was calculated by determining the frequency of GFP signal in I-SceI transfected F-DRGFP cells subtracting the background from nontransfected controls and dividing by the frequency of GFP signal from pGFP transfected F-DRGFP cells. Data shown are the average from three independent experiments, with error bars representing the s.e. (d) Cellular sensitivity to radiation was determined by the loss of colonyforming ability as described in Figure 1. The cells were exposed to X-rays $(4 \mathrm{~Gy})$ at $48 \mathrm{~h}$ after siRNA transfection and were then collected for clonogenic assay. Data shown are the average from three independent experiments with error bars representing the s.e. 